\title{
A search for bioactive peptides in fish mucus
}

\author{
Durita D. Djurhuus $^{\mathrm{a}, \mathrm{b}}$, Magne Sydnes ${ }^{\mathrm{d}, \mathrm{e}}$, Fiona Provan ${ }^{\mathrm{d}}$, Jan Stagsted ${ }^{\mathrm{f}}$, Hóraldur Joensen ${ }^{\mathrm{a}}$, Jonhard Eysturskard ${ }^{\mathrm{c}}$, Svein-Ole Mikalsen $^{\mathrm{a}}$ \\ ${ }^{a}$ Faculty of Natural and Health Sciences, University of the Faroe Islands, Tórshavn, Faroe Islands \\ 'Aquaculture Research Station of the Faroes, Air, Faroe Islands \\ Mathematics and Natural Sciences, University of Stavanger, Stavanger, Nown \\ 'Department of Food Science, Aarhus University, Tjele, Denmark
}

Introduction

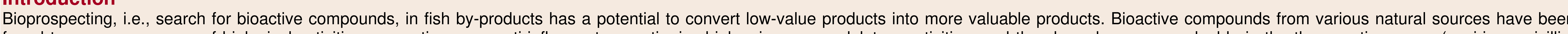

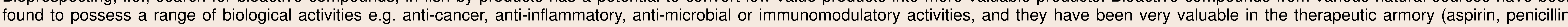

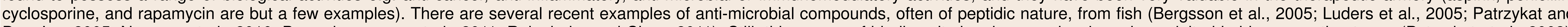

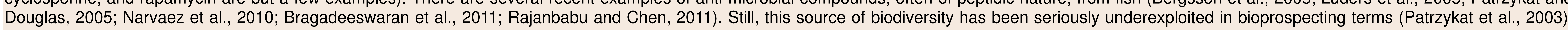
but often overexploited in commercial fisheries. Nations heavily dependent on fishing could create more value from the same amount of catch by bioprospecting the secondary products.

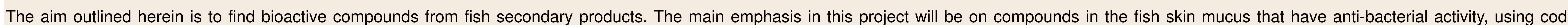

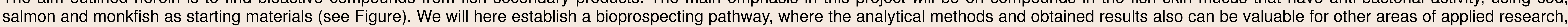
in particular in the fight against salmon (sea) louse (Lepeophtheiris salmonis).

\section{Working plan and methods}

\section{Initial study (first year)}

We will initially focus on the mucus of the fish skin. The mucus is a first-line of defense against the surrounding environment, and interesting bioactive compounds have previously been detected in the mucus from several fish species (Rajanbabu and Chen, 2011) We will make a comparative study of the three species using mass spectrometry (MS) and other proteomic tools to identify as many potentially bioactive compounds as possible.

Identification

As outlined in the figure the bioprospecting pathway includes sampling the mucus and filtration of samples resulting in two initial fractions. Low molecular weight fractions will be directly analyzed by LC-MS/MS. High molecular weight fractions will be subfractionated by isoelectric focusing in a gel free system (Agilent OffGel) and by isoelectric focusing in a gel free system (Agilent OffGel) and further by polyacrylamide electrophoresis. Detec
digested with trypsin and analyzed by LC-MS/MS.

Identification of peptides will first be done by searches against available genome and EST databases. Non-identified peptides with good spectra will be analyzed manually. Some low-molecular weight compounds that are not standard peptides (i.e., do not contains the standard unmodified amino acids) can have biological activities (Falkenberg et al, 2011). These compounds might contain modified (Fakenorger or they might be other classes of molecules. By amino acids, or they might be other classes of molecules. By MS the molecules we will be able to approach the chemical formulas.

Bioactivities

As for investigation of the antibacterial activities, we will first concentrate on the low molecular weight fractions (Rajanbabu and Chen, 2011). The fractions will be tested with a standard bacterial growth assay using OD measurements. We also aim at developing two alternative bacterial assays. The simplest is an assay with poor bacterial growth conditions. Bacteria are much less sensitive to bacterial growth conditions. Bacteria are much less sensitive to antibiotics under poor growth conditions (Eng et al., 1991; Nguyen e al., 2011). Thus, fractions that can kill off bacteria under poor growt condition are even more into account that the envionment usualy contains a mixture of bacteria. We will combine a metagenomic approach for testing of anti-bacteria effects, and investigate if certain bacteria are susceptible to the antibacterial effects while other bacteria are resistant.

Later studies (second and third year)

In the first year studies, we will hopefully have detected bioactivities. The main aim is now to identify the compounds that possess these activities. We will then concentrate on the species that have shown the highest measurable bioactivities in its mucus, and we must develop methods to obtain larger amounts of the mucus. More detailed fractionations will be needed. The initial fractionation methods will be by molecular weight (using filtration, dialysis, size exclusion chromatography, etc.) and by physicochemical properties (preparative HPLC using reverse phase chromatography, ion exchange chromatography, etc.). The results will be analyzed statistically by multivariate analysis and principal component analysis in support of the search for the bioactive compounds.

In cooperation with the Department of Food Science, Aarhus University, it is possible to test for antibacterial and/or immunomodulatory activity using in situ pig intestinal microbial survival model and in vivo infection model. The former model uses the natural intestinal flora of pigs. Immediately after killing a pig the proximal small intestine is exposed, sutured at $10 \mathrm{~cm}$ intervals, and cut to small intestine is exposed, sutured at $10 \mathrm{~cm}$ intervals, and cut to preserve intestinal content and saline. Peptides are added and the intestinal "bags" are incubated a couple of hours. The content is added to bacterial growth media and plated for counting of colonies
the next day. The in vivo infection model uses weaned piglets of the next day. The in vivo infection model uses weaned piglets of a certain genotype that is susceptible to infection with the E. coli strain HB104. The piglets will receive the test material and will
subsequently be inoculated with the E. coli strain. Growth of E. col HB104 and diarrhea will be scored.

Future studies (four to ten years +)

When the structures of the bioactive compounds are at hand, it can be determined whether the best approach for making industrial scale amounts of the compounds are by processing the secondary products from the fish industry, or by synthetic chemistry. Commercialization will be attempted if the compounds are promising

Cod is among the most important catches of wild fish in the North Atlantic region. A proteomic study is available for cod mucus
(Rajan et al., 2011), and some antimicrobia peptides have been found in cod (Bergsson et
al., 2005; Ruangsi et al., 2010). The cod genome is available (Star et al., 2011). In cod, some of the immune elements, which in other species participate din de whe against

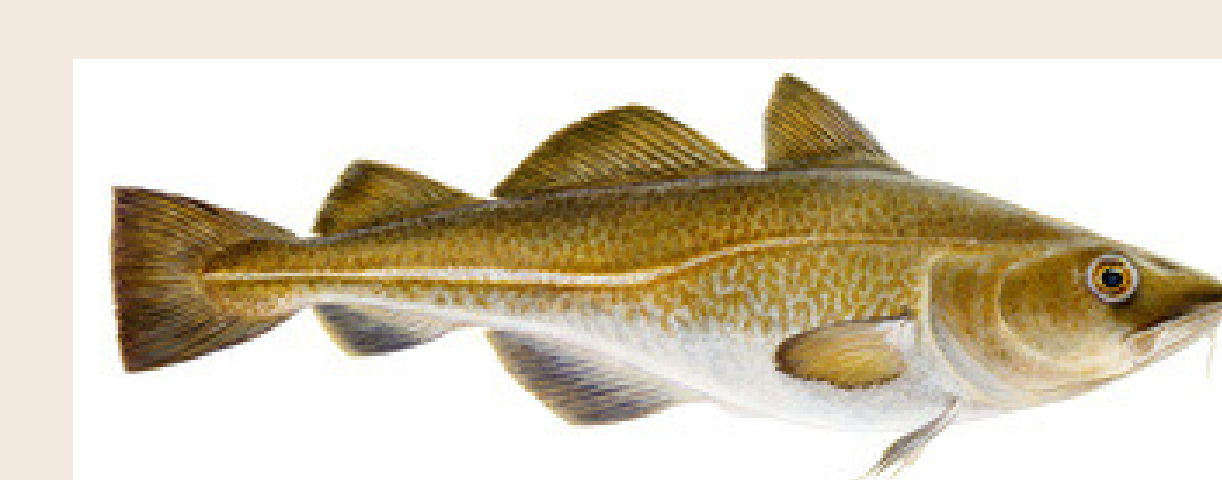

Monkfish is a bottom-dwelling fish, and thus is more or less in constant contact with sea bed strong antimicrobial defense in their mucus. Anecdotes in the Faroe Islands suggest that mucus from monkfish may help wound healing.

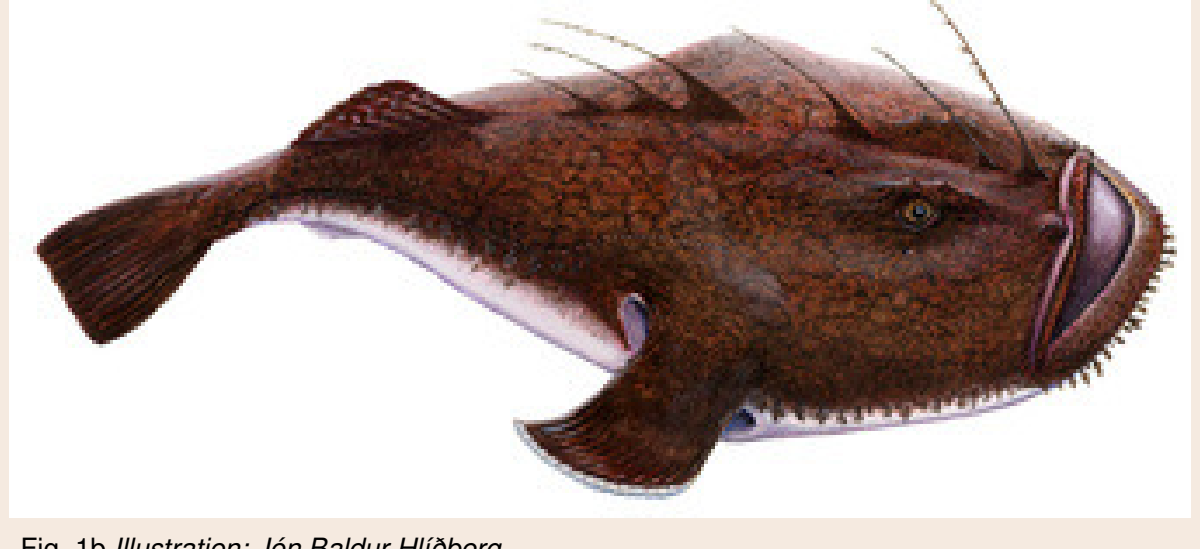

Salmon is the most important farmed fish in

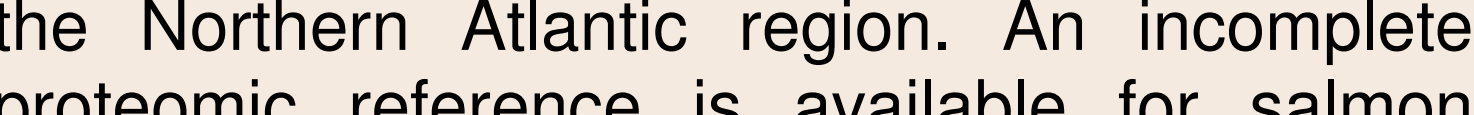
mucus (Easy and Ross, 2009). Antibacterial peptides have been detected in salmon mucus (Luders et al., 2005; Narvaez et al., 2010). Salmon louse (Lepeophtheiris salmonis) attach to the skin of the fish and appear to change the mucus protein compositon (Easy and Ross,

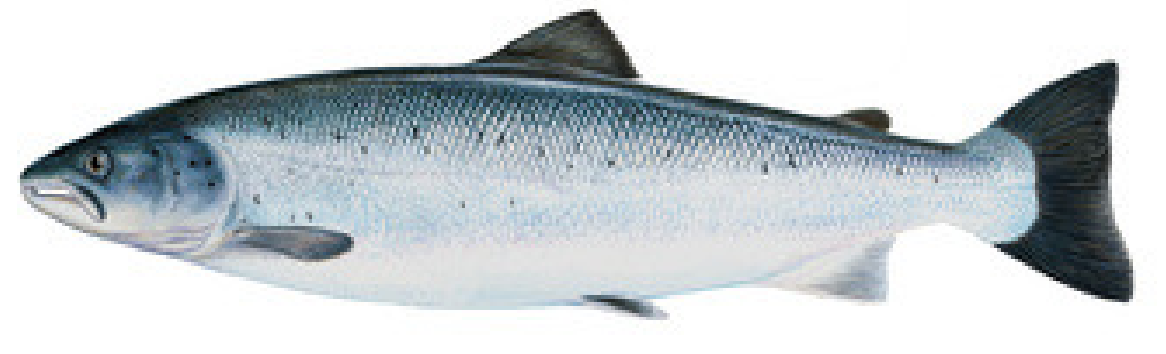

Preliminary study will involve three individuals of each species and four samples will be taken from each fish. Mucus is scraped off the right and left side of the left and right side between the pectoral and pelvic fins using sterile glass slides. In this way, we can compare the constitution of the dorsal and ventral mucus between the three species.

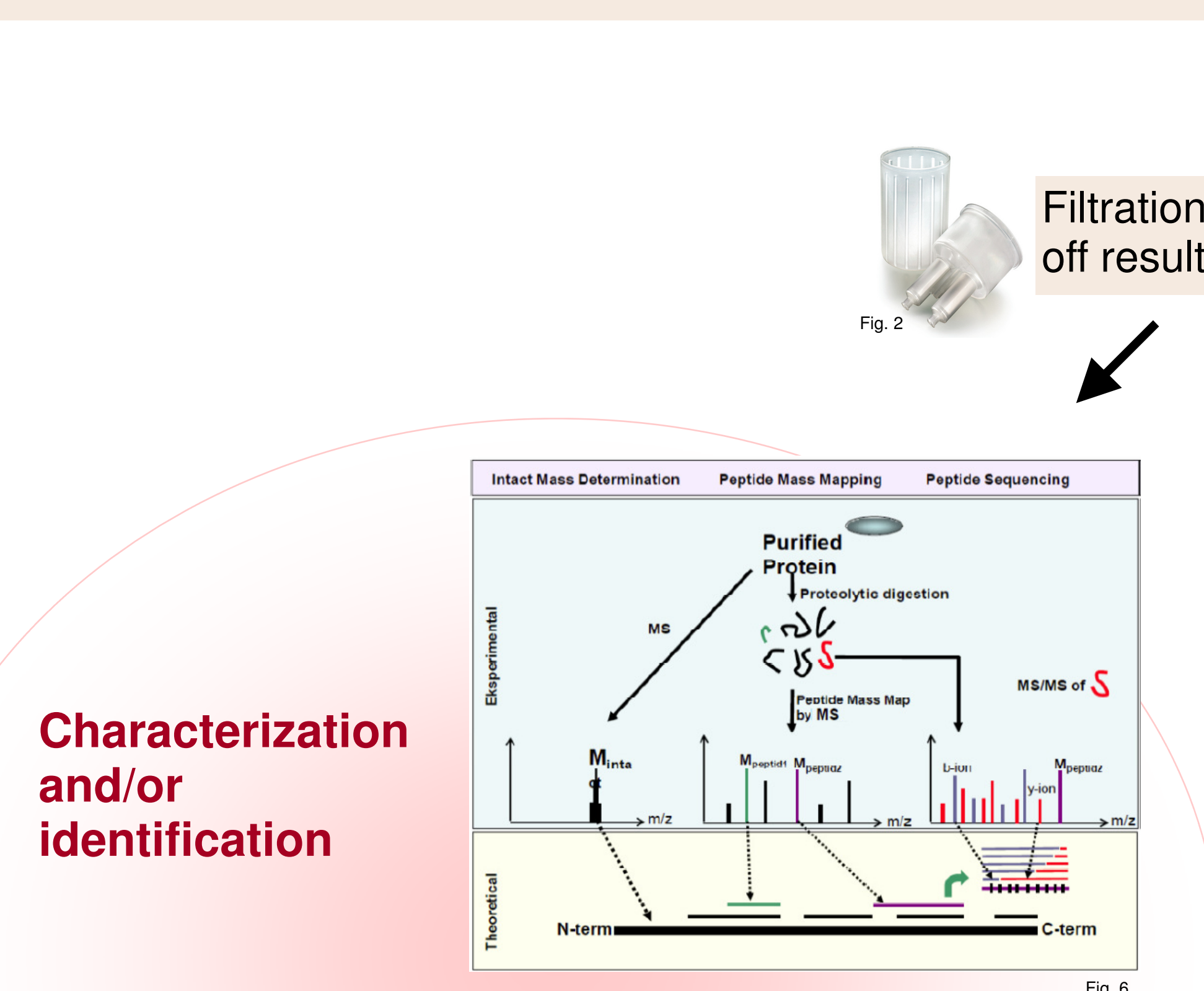

Low molecular weight fractions $(<10 \mathrm{kDa})$ will be directly analysed by LC-MS/MS. High molecular MS/MS after separation and digestion.

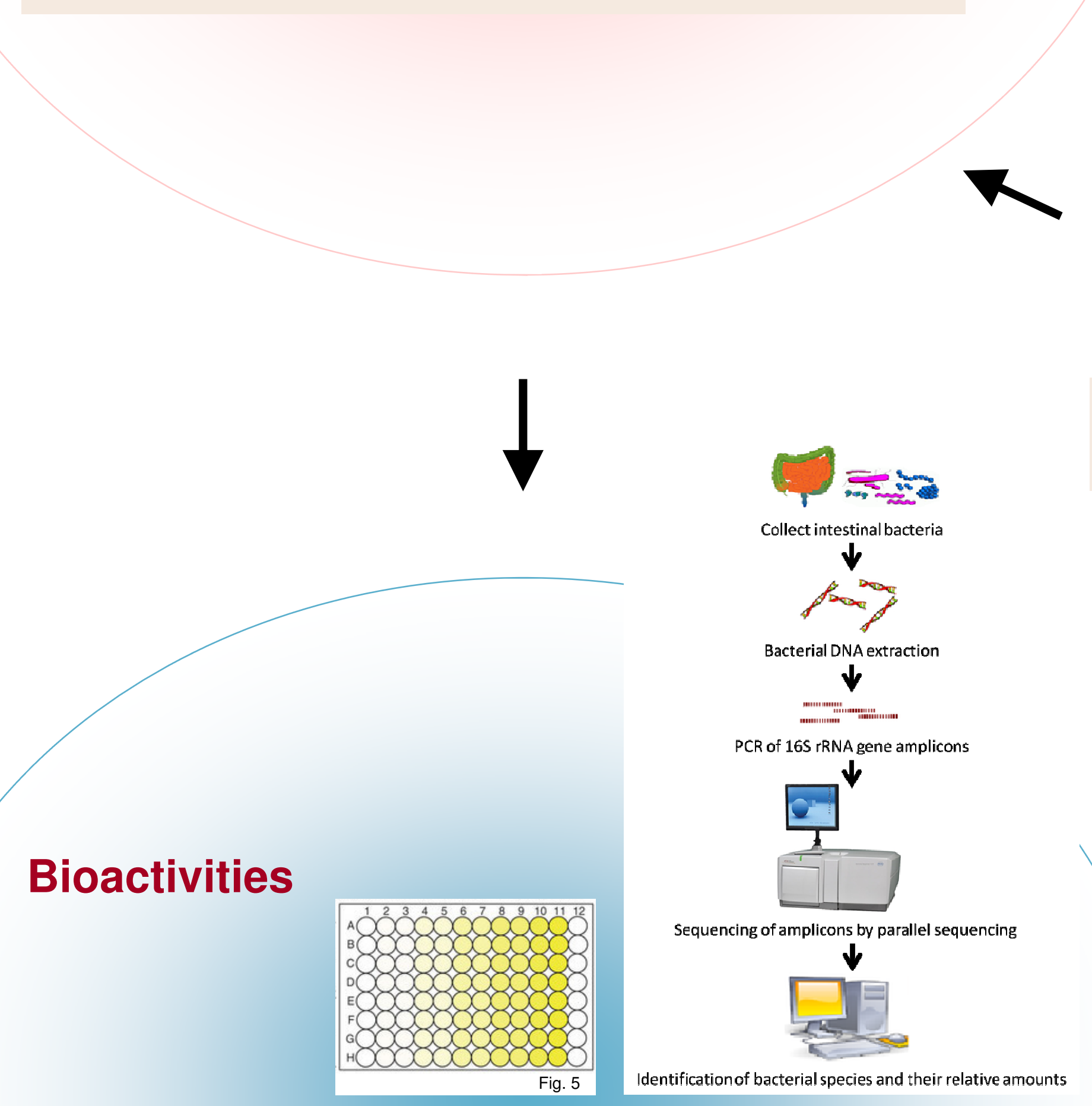

Low molecular weight fractions $(<10 \mathrm{kDa})$ will be directly analyzed by standard bacterial growth assay, an assay with poor bacteria
conditions and by a metagenomic assay.

.

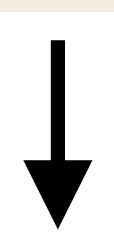

by Centricon filters $10 \mathrm{kDa}$ cutUlting in two fractions.
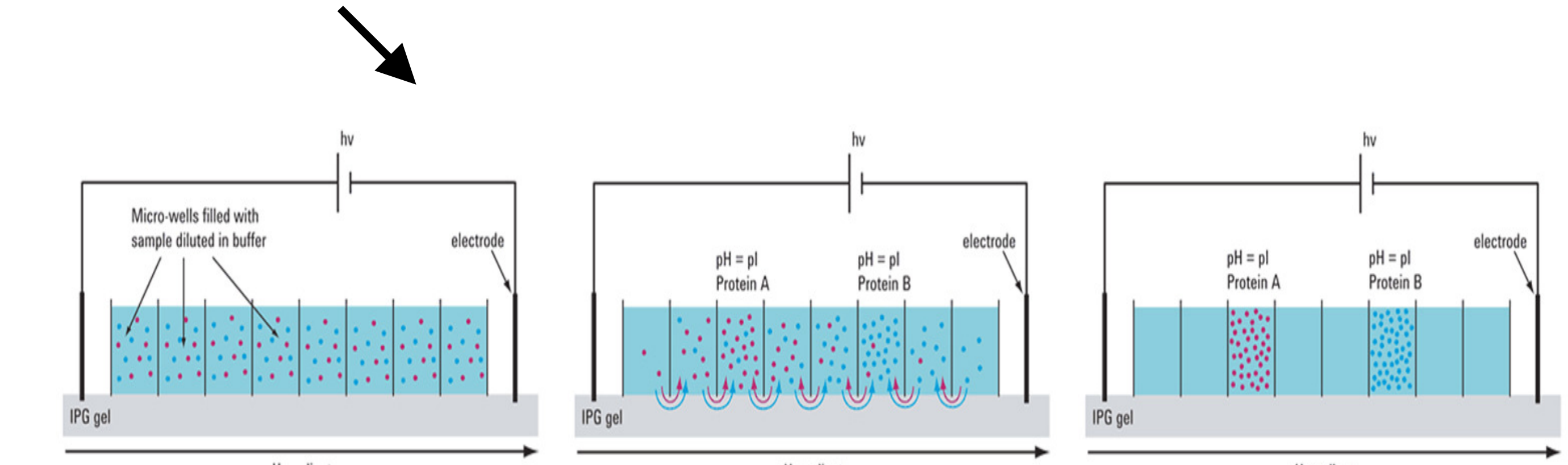

High molecular weight fractions $(>10 \mathrm{kDa})$ will be separated by isoelectric focusing.

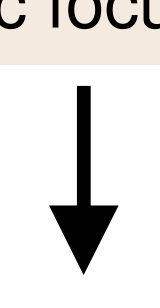

Digestion using trypsin

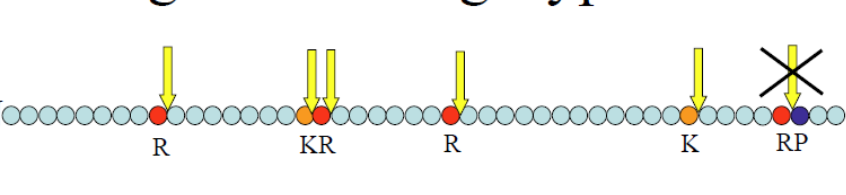

The detected proteins will be digested with trypsin. Furthermore, having established the necessary methods and competence, more complex starting materials can be entered into the bioprospecting pathway.

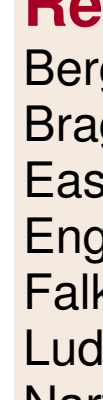

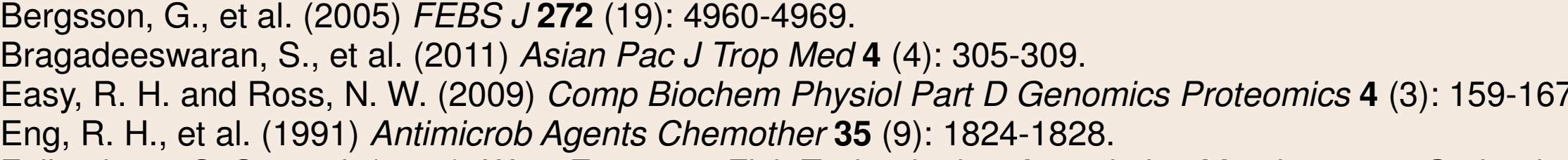

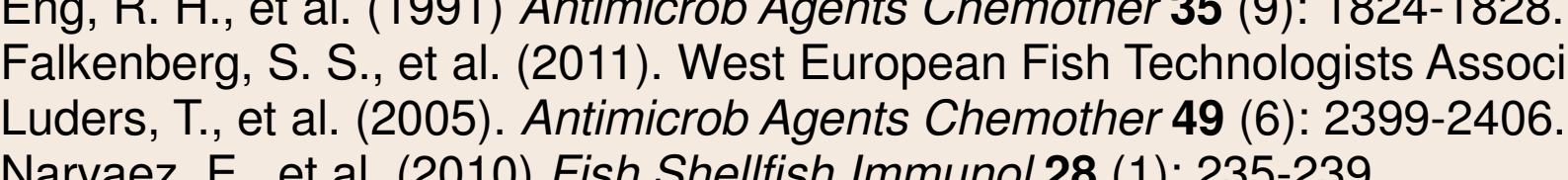

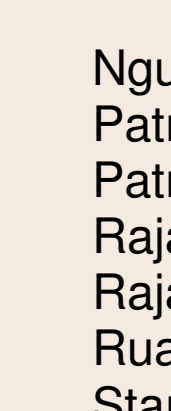

\section{Expected results and effects}

We expect that there should be some differences between bottom-dwelling and pelagic species, as they probably have to be protected against partially different microbial and parasitic environments

Through the work described here, we expect to isolate bioactive compounds, most likely with anti-bacterial activities, from the mucus of fish. The most potent compounds will be structurally determined, and commercialization will be attempted. Furthermore, a thorough characterization of mucus from salmon will also be a cornerstone in the fight against salmon (sea) louse (Lepeophtheiris salmonis). 